\title{
Strangeness production and other recent results from Hall B at Jefferson Lab
}

\author{
R.A. Schumacher ${ }^{\mathrm{a}}$ \\ For the CLAS Collaboration \\ Department of Physics, Carnegie Mellon University, Pittsburgh, PA 15213, USA
}

Received: 30 September 2002 /

Published online: 22 October 2003 - (c) Società Italiana di Fisica / Springer-Verlag 2003

\begin{abstract}
Recent results using the CLAS detector at Jefferson Lab are discussed. We emphasize strangeness photo- and electro-production in the baryon resonance region between $W=1.6$ and $2.3 \mathrm{GeV}$, where indications of $s$-channel structure are suggestive of high-mass baryon resonances coupling to kaons and hyperons in the final state. Other CLAS results, for the $E_{1+} / M_{1+}$ transition form factor at the first $\Delta$-resonance, two-pion electroproduction, and $\eta$ photoproduction are also briefly mentioned.
\end{abstract}

PACS. 13.30.-a Decays of baryons - 13.30.Eg Hadronic decays - 13.40.-f Electromagnetic processes and properties - 13.60.-r Photon and charged-lepton interactions with hadrons

\section{Introduction}

The CLAS detector at Jefferson Lab [1-5] serves as a large-acceptance, moderate-momentum resolution facility for probing nucleon excitations, meson production, and correlations in light nuclear systems. This presentation features highlights from a series of measurements dedicated to the study of open strangeness systems in the baryon resonance region. New results for both photo- and electro-production of strangeness-containing final states will be discussed. Complementary work in resonance excitation in the non-strange final states will be mentioned and the reader will be referred to the recent literature.

Baryon resonances are a basic problem of interest in non-perturbative QCD. The masses, quantum numbers, and decay branches of the higher-mass baryon resonances have remained difficult to establish, both experimentally and theoretically. Experimentally, most of what we know comes from the use of hadron beams, most commonly pions, interacting with nucleon targets, followed by detection of one or more pions plus the nucleons in the final state. At higher energies, both the energy overlap problem, and, above the two-pion threshold, $\rho$-meson production, make it more difficult to separate the resonance contributions. The large number of poorly established higher-mass resonances listed by the Particle Data Group [6] attests to the challenge of doing accurate work above the strangeness threshold near $1600 \mathrm{MeV}$. Theoretically, there is the well-

\footnotetext{
${ }^{a}$ e-mail: schumacher@cmu.edu
}

known over-supply of baryons predicted in quark models, the so-called "missing baryons" problem [7], and various suggested ways that dynamical effects such as di-quarks could prune the spectrum to something close to what has been observed [8].

Electromagnetic production of resonances that decay into strange particles offers two advantages. First, twobody $K Y$ final states are experimentally easier to analyze than the three-body $\pi \pi N$ final states that dominate decays at higher masses. So, while the cross-sections for strangeness production tend to be small (on the order of 1 or $2 \mu \mathrm{b}$ in electromagnetic production) there can be a pay-off in terms of ease of interpretation of energy and angular distributions. Second, couplings of resonances to strangeness-containing final states are expected to be different than coupling to pionic final states [7]. Therefore, the strangeness sector casts a different light on the resonance excitation spectrum, and thus offers an opportunity for seeing resonances not revealed clearly using pions.

It must be added, however, that to date the PDG compilation lists only five well-established resonances with poorly known coupling to the $K \Lambda$ final state, and none with couplings to the $K \Sigma^{0}$ final state. The most widely available theoretical calculation of the electromagnetic $K \Lambda$ production, the Kaon-MAID code [9], includes a mere three well-known resonances: the $S_{11}(1650)$, the $P_{11}(1710)$, and the $P_{13}(1720)$. Thus it is timely and interesting to have additional good-quality photo- and electroproduction data of these channels to see what additional resonance formation and decay information can be found. 


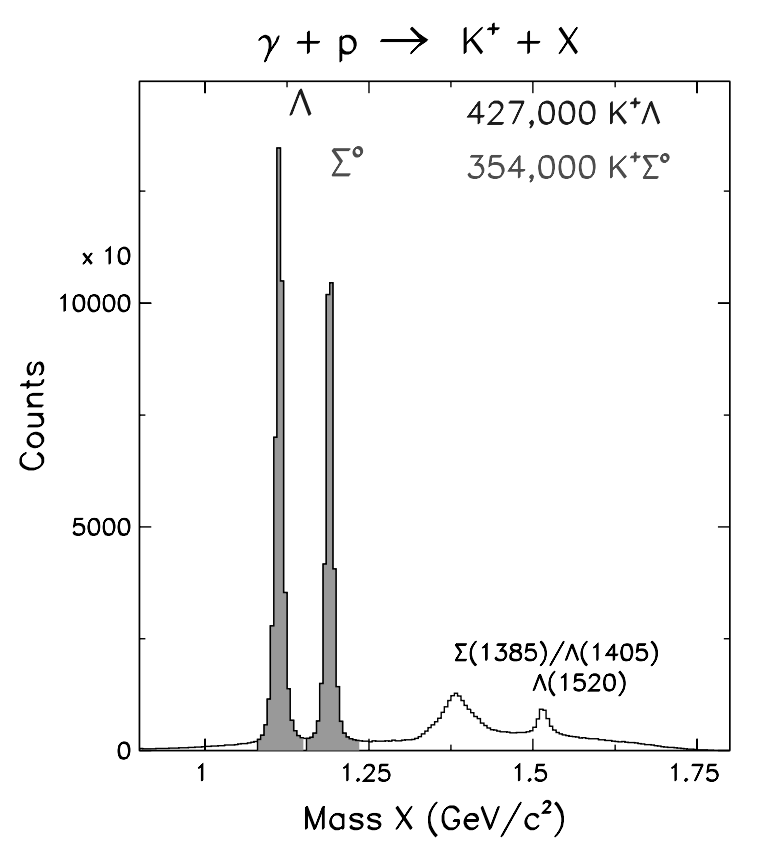

Fig. 1. Missing-mass plot for the CLAS photoproduction data set with $2.4 \mathrm{GeV}$ endpoint energy, summed over all kaon detection angles.

\section{$2 \mathrm{KY}$ photoproduction}

Figure 1 shows the missing-mass spectrum obtained with CLAS for the real photon reaction $\gamma+p \rightarrow K^{+}+X$ after kaon selection cuts. The beam of tagged photons had an electron endpoint energy of $2.4 \mathrm{GeV}$, and spanned all photon energies from threshold at $E_{\gamma}=0.911 \mathrm{GeV}$ up to $2.3 \mathrm{GeV}$. A mass resolution of $\sigma=6.1 \mathrm{MeV}$ was obtained when averaging over all detection angles and photon energies. The $\Lambda$ and $\Sigma^{0}$ events were separated from the background of mis-identified pions using lineshape fits to missing-mass spectra in each bin of photon energy and kaon angle.

Our results were binned in $25 \mathrm{MeV}$ steps in $W$ and in 20 bins in the center-of-mass angle of the kaons, $\cos \left(\theta_{K}^{\text {c.m. }}\right)$. CLAS has acceptance holes in the forward and backward directions for $\cos \left(\theta_{K}^{\text {c.m. }}\right)>0.9$. A Legendre polynomial moment analysis of the angular distributions was used to extrapolate into these regions, causing a source of systematic uncertainty into the total cross-section results shown here. The full analysis of the angular distributions will be discussed in a future publication.

Figure 2 shows the total cross-section for $\Lambda$-hyperon photoproduction. Overall there is fair agreement between CLAS and previous data from SAPHIR at Bonn [11]. The CLAS data are preliminary in that we are still working on $10 \%$-level corrections to the normalization and acceptances. Most notable above the threshold region where the known $S_{11}, P_{11}$, and $P_{13}$ resonances dominate, is the broad unexplained structure centered at $1.9 \mathrm{GeV}$. This structure, first seen in the Bonn data, was interpreted by Mart and Bennhold [10] and collaborators as evidence for a "missing" resonance at this mass. Based on theoretical considerations from a particular quark model [7], an

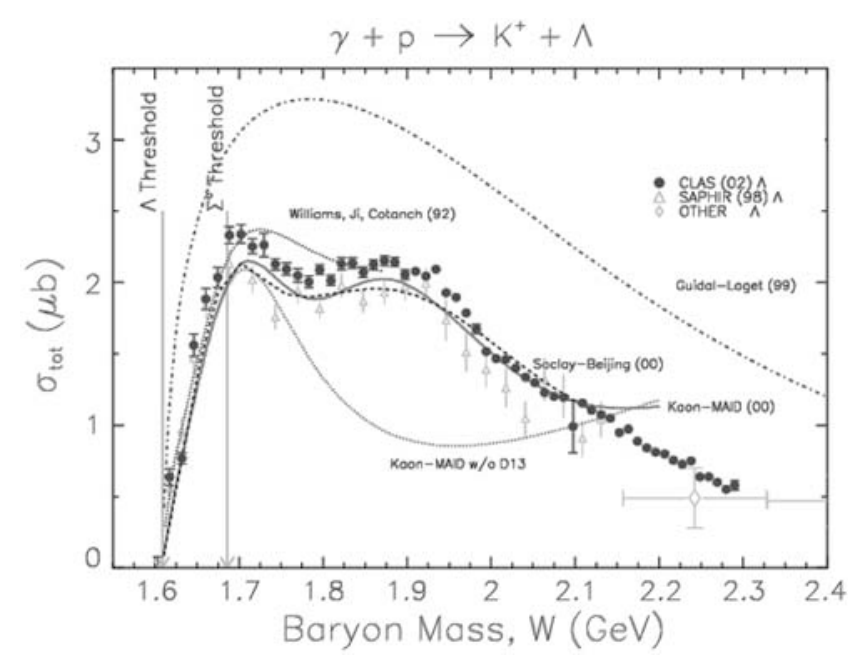

Fig. 2. Total cross-section for $\Lambda$-hyperon photoproduction measured at CLAS (solid circles). Data from SAPHIR/Bonn [11] (open triangles) are also shown. The curves are for effective Lagrangian calculations of Bennhold et al. as computed by Kaon-MAID [9] (solid), Williams, $\mathrm{Ji}$, and Cotanch [12] (upper dotted), Saghai [13] (dashed), and a Regge-model calculation of Guidal et al. [14,15] (dotdashed). The lower dotted curve illustrates the effect when the $D_{13}(1900)$ resonance in the Kaon-MAID calculation is switched off.

assignment of $D_{13}(1900)$ seemed consistent with the angular distributions. However, other groups showed that the same data could be accommodated either with $u$-channel exchanges [13] or with an extra $P$-wave resonance [16]. We expect the CLAS data to resolve this question when the full angular distributions are released.

The Regge-model calculation $[14,15]$ shown in fig. 2 is too high by a substantial factor for this channel. The calculation uses only $K$ and $K^{*}$ exchanges, with no resonances at all. The prediction was made using a model that fit high-energy kaon electroproduction data very well. However, extrapolated into the resonance region, the model fails. As seen below, it behaves much more as expected in the case of $\Sigma^{0}$ production.

In fig. 3 the total cross-section for the $\Sigma^{0}$-hyperon is shown. In this case, both isospin- $(1 / 2)$ and isospin- $(3 / 2)$ nucleon resonances can contribute. Here there is a peak in the total cross-section near $1.9 \mathrm{GeV}$, which has in the past been associated with the cluster of $\Delta$-resonances in this mass range. As mentioned earlier, no such resonances have been reliably shown to have decay branches to strange final states. These data, as do the previous Bonn data, clearly show the imprint of resonant decays to $K \Sigma^{0}$.

With an unpolarized photon beam and an unpolarized target, hyperons can still be produced polarized along the axis perpendicular to the production plane. This is known as the "recoil" polarization. The large acceptance of CLAS made it straightforward to detect protons from the decay of hyperons in coincidence with the K-mesons. The well-known parity-violating weak decay asymmetry in hyperon decays permits us to determine this polarization by measuring the angular distribution of the decay protons. 


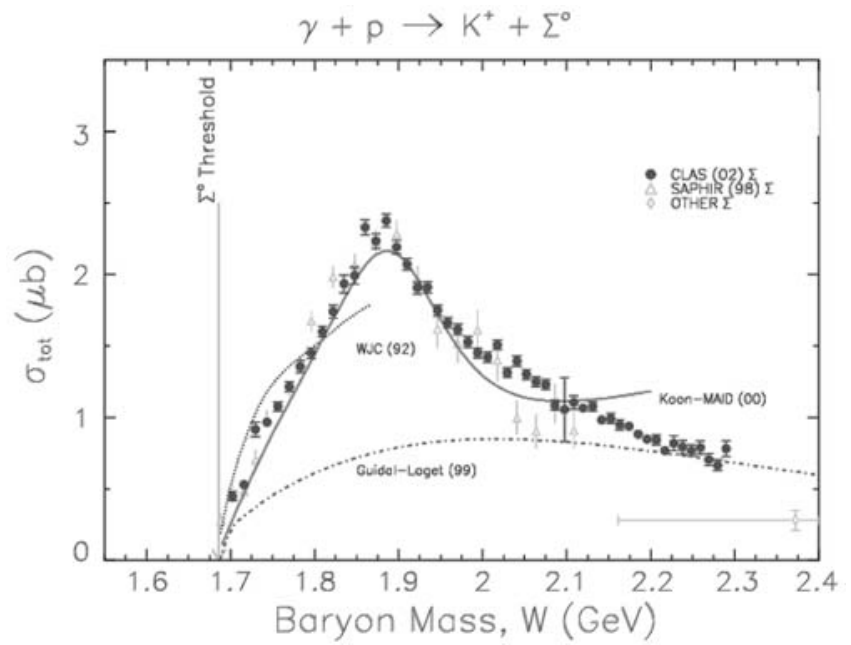

Fig. 3. Total cross-section for $\Sigma^{0}$-hyperon photoproduction measured at CLAS (solid circles). Data from SAPHIR/Bonn [11] (open triangles) are also shown. The curves are for effective Lagrangian calculations of Bennhold et al. [17] as computed by Kaon-MAID [9] (solid), Williams, Ji, and Cotanch [12] (dotted), and a Regge-model calculation of Guidal et al. $[14,15]$ (dot-dashed).

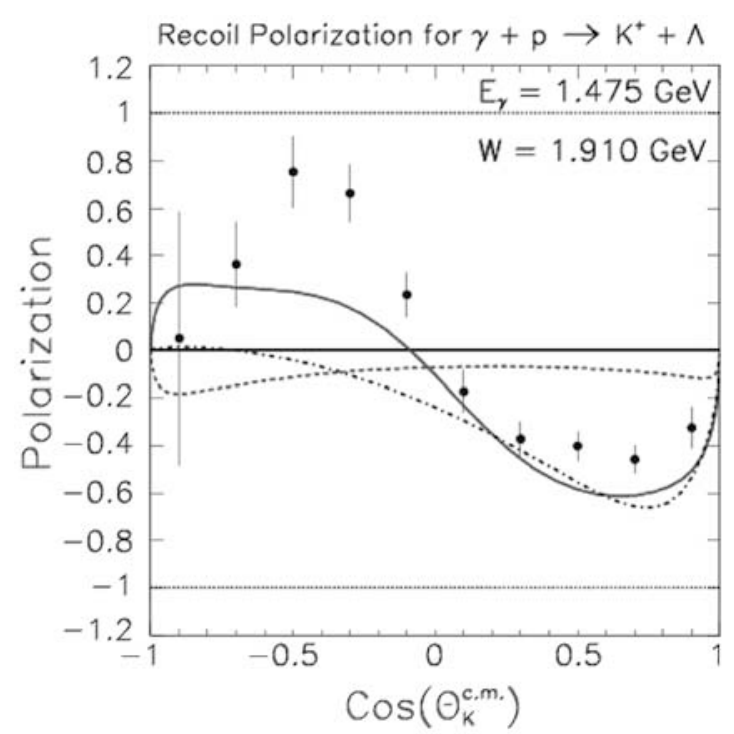

Fig. 4. Polarization of the $\Lambda$-hyperon as a function of kaon center-of-mass production angle for a total system mass, $W$, of $1.90 \mathrm{GeV}$. For comparison, the curves are for the hadrodynamic calculation of Bennhold et al. [17] as computed by KaonMAID [9] (full), and a Regge-model calculation of Guidal et al. $[14,15]$ (dot-dashed). The dashed curve illustrates the effect when the $D_{13}(1900)$-resonance in the Kaon-MAID calculation is switched off.

Measurement of this observable is interesting since it is related to interferences of the imaginary parts of the resonant amplitudes with other amplitudes, including Born terms.
In fig. 4 we show an example of the data obtained at CLAS, for an energy bin at the peak of the broad $K \Lambda$ cross-section rise, $W=1.9 \mathrm{GeV}$, and for the full range of kaon angles in the c.m. frame. We see strong negative polarization of the $\Lambda$ hyperons when the kaons are in the forward direction, and an equally strong positive polarization when the kaons go backward. Our results are consistent with a few older data points from Bonn [11], although they had no data at this particular energy. It is interesting to see that the MAID calculation is very sensitive to the presence of the $D_{13}(1900)$ (dashed curve), for indeed this is the only resonant amplitude included in this calculation in this mass range. This observation is in contradiction to the statement made in ref. [10] when discussing the MAID model in relation to the Bonn data. It is clear that the MAID model, while providing some back-angle recoil polarization via a $D_{13}$ at $1900 \mathrm{MeV}$, still misses the measured polarization by a factor of 2 at some angles. The Regge model is not able to provide any back-angle polarization, since by construction it has only a $t$-channel dominated (forward-angle) production mechanism. CLAS has polarization data of the sort shown in fig. 4 for the full range of energies shown in fig. 2, but final systematic checks are still in progress.

\section{$3 \mathrm{KY}$ electroproduction}

Moving away from the 4-momentum transfer $Q^{2}=0$ limit of photoproduction, CLAS has measured exclusive $K^{+} \Lambda$ and $K^{+} \Sigma^{0}$ electroproduction on the proton for $0.5<$ $Q^{2}<1.1 \mathrm{GeV} / c^{2}$ at a beam energy of $2.6 \mathrm{GeV}$. For unpolarized electrons and target, and after dividing out the flux of virtual photons in a given bin of $W$ and $Q^{2}$, the crosssection for pseudo-scalar meson electroproduction reads:

$$
\begin{aligned}
\frac{\mathrm{d} \sigma_{\gamma}}{\mathrm{d} \Omega_{K}}= & \sigma_{\mathrm{T}}+\epsilon \sigma_{\mathrm{L}}+\epsilon \sigma_{\mathrm{TT}} \cos (2 \phi) \\
& +\sqrt{2 \epsilon_{\mathrm{L}}(\epsilon+1)} \sigma_{\mathrm{LT}} \cos (\phi),
\end{aligned}
$$

where $\epsilon$ is the polarization parameter of the virtual photon, and $\phi$ is the azimuthal angle between the electron-scattering $\left(e, e^{\prime}\right)$ and the hadronic $(K Y)$ planes. Exploiting the $\phi$-dependence of the process allows one to pick out the interference cross-sections at a given $Q^{2}$, $W, \cos \left(\theta_{K}^{\text {c.m. }}\right)$, and $\epsilon$, or, using the Rosenbluth technique, separating the transverse $\left(\sigma_{\mathrm{T}}\right)$ and longitudinal $\left(\sigma_{\mathrm{L}}\right)$ cross-sections.

At CLAS we have determined the so-called unseparated transverse and longitudinal cross-sections $\left(\sigma_{\mathrm{T}}+\epsilon \sigma_{\mathrm{L}}\right)$ and, for the first time in the resonance region, the interference cross-sections $\sigma_{\mathrm{LT}}$ and $\sigma_{\mathrm{TT}}$. The $W$ range was the same as in photoproduction. At the amplitude level, the electron scattering interference responses $\sigma_{\mathrm{LT}}$ and $\sigma_{\mathrm{TT}}$ are related to real photon measurements of polarized beam asymmetries, and so they are sensitive to some of the same structure information [18].

In fig. 5 we show the missing-mass spectrum for hyperon electroproduction, again summed over all of CLAS, 


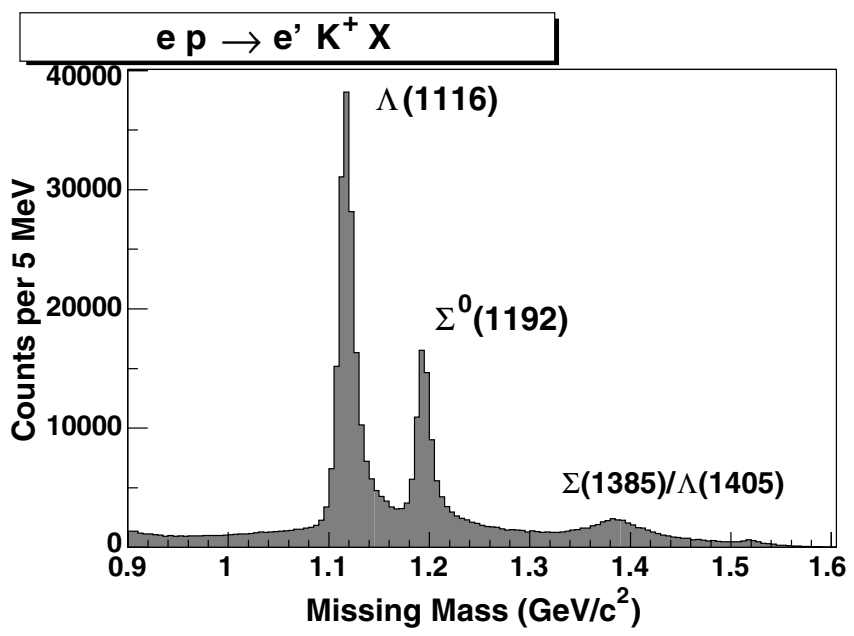

Fig. 5. Missing-mass plot for the CLAS data set with $2.6 \mathrm{GeV}$ electron energy, summed over all kaon detection angles. The resolution is $7.5 \mathrm{MeV}$. The background, due to mis-identified pions, was removed via line-shape fitting that included radiative effects.

and for the full range of $Q^{2}$ that was measured. The resolution is similar to the real photoproduction case shown in fig. 1 . The radiative tails can be seen on the high-mass sides of the hyperon peaks. After dividing into bins according to the kinematic variables in the problem $\left(W, Q^{2}\right.$, $\left.\cos \left(\theta_{K}\right), \phi\right)$, the missing-mass spectra were fit to individually calculated lineshapes that included pion background.

In fig. 6 we show the $\phi$-dependence of the cross-section for $\Lambda$ and $\Sigma^{0}$ electroproduction in one specific bin, near $W=1.9 \mathrm{GeV}$, where in photoproduction there is a broad maximum in the cross-section. One sees that the interference behavior is very different: $\sigma_{\mathrm{LT}}$ for the two hyperons will have opposite signs, and $\sigma_{\mathrm{TT}}$ for the $\Lambda$ is small while for the $\Sigma^{0}$ it is large. The kinematic dependence of the cross-section structure functions show that $\Lambda^{\prime}$ s and $\Sigma^{0}$ 's are produced very differently. $t$-channel processes seem to dominate $\Lambda$ production, with large contributions from the longitudinal part of the interaction. Production of the $\Sigma^{0}$-hyperon is more $s$-channel resonance dominated. The cross-section results are not quite final enough for inclusion here.

\section{Other CLAS results}

Space does not permit an extensive review of the other CLAS results discussed in this presentation. In several cases the results are now available elsewhere. The CLAS results for $E_{1+} / M_{1+}$ for the $N \rightarrow \Delta$ transition have been published [19] and will not be reproduced here.

Recent measurements of $\eta$ photoproduction have been made that extend the differential and total cross-section measurements up to $W=2.13 \mathrm{GeV}$, well above the previous limits defined by experiments at Mainz and GRAAL. These results have been submitted for publication and are available [20].
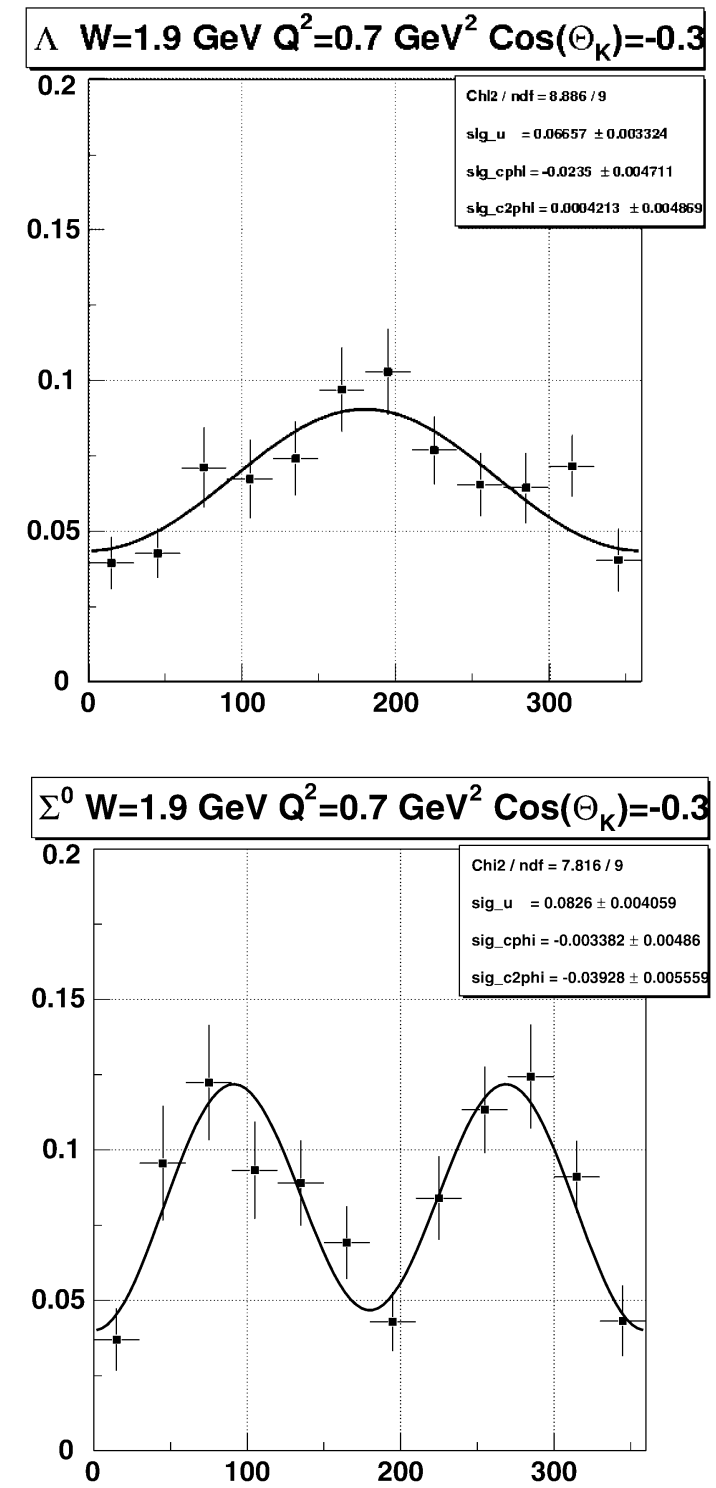

Fig. 6. Dependence of the hyperon electroproduction crosssections on $\phi$ in a given kinematic bin: $W=1.900 \pm 0.025 \mathrm{GeV}$, $Q^{2}=0.7 \pm 0.2(\mathrm{GeV} / c)^{2}$, and $\cos \left(\theta_{K}^{\text {c.m. }}\right)=-0.3 \pm 0.1$ The vertical scales are arbitrary. The fits are of the form $A+B \cos (\phi)+C \cos (2 \phi)$.

CLAS data for electroproduction of two-pion and of $\omega$ meson final states show some evidence [21] for new structure in the higher-mass baryon resonance excitation region. Collectively, the new results from CLAS indicate that we have entered a new era of experimental progress in the baryon resonance problem.

Work on finalizing the hyperon cross-sections and polarizations shown here should be completed by the end of 2002. The main qualitative conclusion is already clear: these data show significant unexplained baryon resonance structure at higher masses. New amplitude-level analyses are called for to more fully unravel the structure of hyperon photo- and electro-production. 


\section{References}

1. W. Brooks et al., Nucl. Phys. A 663, 1077 (2000).

2. M.D. Mestayer et al., Nucl. Instrum. Methods A 449, 81 (2000).

3. E.S Smith et al., Nucl. Instrum. Methods A 432, 265 (1999).

4. G. Adams et al., Nucl. Instrum. Methods A 465, 414 (2001).

5. A. Amarian et al., Nucl. Instrum. Methods A 460, 239 (2001).

6. Particle Data Group, Phys. Rev. D 66, 1 (2002).

7. Simon Capstick, W. Roberts, Phys. Rev. D 58, 1 (1998), and references therein.

8. See for example: E. Klempt, nucl-ex/0203002.

9. T. Mart, C. Bennhold, H. Haberzettl, L. Tiator, KaonMAID 2000 at www.kph.uni-mainz.de/MAID/kaon/ kaonmaid.html.

10. T. Mart, C. Bennhold, Phys. Rev. C 61, 012201 (2000).

11. M.Q. Tran et al., Phys. Lett. B 445, 20 (1998); C. Bennhold et al., nucl-th/9901066; M. Bockhorst et al., Z. Phys. C 63, 37 (1994).

12. Robert A. Williams, Cheung-Ryong Ji, Stephen R. Cotanch, Phys. Rev. C 46, 1617 (1992); see also Stephen Cotanch, Shian Hsiao, Nucl. Phys. A 450, 419 (1986)c.
13. B. Saghai, private communication. See also: J.C. David, C. Fayard, G.H. Lamot, B. Saghai, Phys. Rev. C 53, 2613 (1996).

14. M. Guidal, J.-M. Laget, M. Vanderhaegen, Nucl. Phys. A 627, 645 (1997).

15. M. Vanderhaegen, M. Guidal, J.-M. Laget, Phys. Rev. C 57, 1454 (1998).

16. S. Janssen, J. Ryckebusch, D. Debruyne, T. Van Cauteren, Phys. Rev C 65, 015201 (2001); S. Janssen, J. Ryckebusch, W. Van Nespen, D. Debruyne, T. Van Cauteren, Eur. Phys. J. A 11, 105 (2001).

17. C. Bennhold, H. Haberzettl, T. Mart, in Proceedings of the 2nd ICTP International Conference on Perspectives in Hadron Physics, Trieste, Italy, 10-14 May 1999, edited by S. Boffi, C. Ciofi degli Atti, M. Giannini (World Scientific, Singapore, 2000), nucl-th/9909022.

18. See for example: G. Knoechlein, D. Drechsel, L. Tiator, Zeit. Phys. A 352, 327 (1995).

19. K. Joo et al., Phys. Rev. Lett. 88, 122001 (2002).

20. M. Dugger et al., Phys. Rev. Lett. 89, 222002 (2002), www.public.asu.edu/ britchie/etaPRL.pdf.

21. M. Ripani et al., in preparation. 